VOL. $14(1976), 63-70$.

\title{
A note on locally finite varieties
}

\section{John S. Wilson}

Evidence is presented which suggests that the following assertions about a variety $\underline{\underline{V}}$ of groups may be equivalent:

(a) $\underline{\underline{V}}$ is locally finite,

(b) all V-groups satisfying the maximal condition for normal subgroups are finite, and

(c) all V-groups satisfying the minimal condition for normal subgroups are finite.

\section{Introduction}

In this note we are concerned with three conditions which may be satisfied by a variety $\underline{\underline{V}}$ of groups:

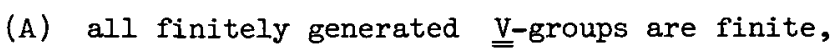

(B) all $\underline{V}$-groups satisfying $\max -n$ are finite, and

(C) all V-groups satisfying min-n are finite.

Here, $\max -n$ and $\min -n$ denote respectively the maximal condition and the minimal condition for normal subgroups.

Condition (A) simply expresses the fact that $\underline{\underline{V}}$ is a locally finite variety, and it is not hard to see that each of (B) and (C) implies that $\underline{\underline{V}}$ has finite exponent. It seems possible that (A), (B), and (C) may be equivalent, and therefore that $(B)$ and $(C)$ may provide characterizations of locally finite varieties. Our object here is to provide some evidence in support of this statement.

Received 30 October 1975. The author is grateful to the Australian National University for a Visiting Fellowship, during the tenure of which this note was prepared. 
Let us begin by observing that (B) implies (A). Suppose that a variety $\underline{\underline{V}}$ contains an infinite finitely generated group $G$. A wellknown result of Baer ( $[1], \S I$, Lemma 1) shows that $G$ has an infinite homomorphic image $\bar{G}$ all of whose proper homomorphic images are finite; and because $\bar{G}$ clearly satisfies $\max -n$, the variety $\underline{\underline{V}}$ cannot satisfy (B).

Further information connecting (A), (B), and (C) is unfortunately less complete: we need to impose (possibly vacuous) conditions on the varieties under consideration. We state our main results as follows:

THEOREM 1. (a) If $\mathrm{V}$ is a locally finite variety containing only finitely many isomorphism classes of finite simple groups, then every

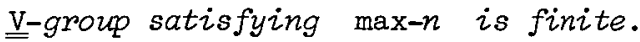

(b) If $\mathrm{V}$ is a locally finite variety containing no infinite simple groups, then every $\underline{\underline{V}-g r o u p ~ s a t i s f y i n g} \min -n$ is finite.

THEOREM 2. Suppose that $\underline{\mathrm{V}}$ is a variety of finite exponent satisfying the following three conditions:

(i) $\underline{\underline{V}}$ contains no infinite finitely generated simple groups,

(ii) $\underline{\underline{V}}$ contains only finitely many isomorphism classes of finite simple groups, and

(iii) for each integer $r$ and prime $p$ there is a bound on the orders of finite r-generator p-groups in $\underline{\underline{V}}$.

Then $\underline{\underline{\mathrm{V}}}$ is locally finite.

In particular, any variety $\underline{\underline{V}}$ satisfying ( $\mathrm{C}$ ) and conditions (ii) and (iii) is locally finite.

It follows from Theorem 6 of Kargapolov [3] that any infinite simple locally finite group involves infinitely many non-isomorphic finite simple groups. Further, for each integer $n$, there are among the known finite simple groups only finitely many isomorphism classes of groups of exponent $n$. It is therefore plausible to suppose that the conditions on finite simple groups in Theorem $I(a)$ and Theorem 2 and on infinite simple groups in Theorem 1 (b) are vacuous; indeed, unless (A) and (B) are equivalent and imply (C), not only are there infinitely many unknown finite simple groups, but also finite simple groups requiring arbitrarily many 
generators. The conjecture is, of course, that every finite simple group is a 2-generator group.

If we combine Theorem $I$ with the result of Walter [6] that all finite simple groups with abelian 2-subgroups are known (up to the determination of the groups of Ree-type), and with Thompson's result that all finite simple groups of order prime to three are Suzuki groups, we obtain

COROLLARY 1. If $\underline{\underline{\mathrm{V}}}$ is a locally finite variety of exponent not divisible by 12 , then any $\underline{\mathrm{V}}$-group satisfying $\max -n$ or $\min -n$ is finite.

The condition on $p$-groups in Theorem 2 is of course the requirement that the restricted Burnside problem should have a positive solution for V-groups of prime-power exponent, and from the main result of Kostrikin [4], this will be the case if $\underline{\underline{V}}$ has square-free exponent. Thus we have

COROLLARY 2. Let $\underline{\underline{\mathrm{V}}}$ be a variety of finite square-free exponent. If $\underline{\underline{V}}$ contains no infinite finitely generated simple groups, then $\underline{\underline{V}}$ is locally finite.

So, at least for varieties $\underline{\underline{V}}$ of square-free exponent, conditions (A), (B), and (C) are equivalent.

Conditions similar to $(A),(B)$, and $(C)$ may be studied in which the role of the class of finite groups is played by some other class $\underline{\underline{X}}$ of groups. Classes $\underline{\underline{X}}$ of finite groups may be chosen to avoid the difficulties with finite simple groups encountered above. An implication of type $(B) \Rightarrow(A)$ holds for any class $\underline{\underline{X}}$ of finitely presented groups. It is easy to show that a variety is locally nilpotent if and only if its groups satisfying $\max -n$ are nilpotent, and that its groups satisfying min- $n$ are then also nilpotent. It would be interesting to know what happens for other classes $\underline{\underline{X}}$ : whether, for example, a variety is necessarily metabelian if all of its groups satisfying $\max -n$ are metabelian.

\section{Proofs}

We begin.by proving two lemmas; the first of these is an extension of a result in Wilson [9].

LEMMA 1. Let $G$ be a locally finite group of (finite) exponent 
dividing $e$, and let $p$ be a prime dividing $e$. If $H$ is a subnormal subgroup of $G$ and if $H$ has no non-trivial locally $p$-soluble homomorphic images, then $H$ is normalized by the group $G^{m}$ generated by all mth powers of elements of $G$, where $m=p^{-1} e$.

Proof. For $G$ finite, this follows from Theorem A of Wilson [9]. We therefore suppose $G$ infinite, and choose an element $x$ of $H$ and an element $y$ of $G$; we must show that $x^{z} \in H$, where $z=y^{m}$. For each finite group $X$ we write $X^{*}$ for the least normal subgroup of $X$ with $p$-soluble quotient group $X / X^{*}$. It is easy to see that $X_{1} \leq X_{2}$ implies $X_{1}^{*} \leq X_{2}^{*}$ and that the subgroups

$$
\left\{X^{*} ; X \text { finite, } X \leq H\right\}
$$

form a local system for a normal subgroup $H^{*}$ of $H$ such that $H / H^{*}$ is locally $p$-soluble. We conclude that $H=H^{*}$, and that there is a finite subgroup $X_{1}$ of $H$ with $x \in X_{1}^{*}$. We set $X=\left\langle y, X_{1}\right\rangle$. Because $(H \cap X)^{*}$ is a subnormal subgroup of the finite group $X$, the case of Lemma 1 in which $G$ is finite implies that $(H \cap X)^{*}$ is normalized by z. But

$$
x \in X_{1}^{*} \leq(H \cap X)^{*},
$$

so that $x^{z} \in(H \cap X)^{*} \leq H$, and Lemma 1 follows.

our second lemma concerns a situation arising in the proofs of both Theorem $I(a)$ and Theorem 2.

LEMMA 2. Let $G$ be an infinite group of finite exponent. If alz proper homomorphic images of $G$ are finite, and if $G$ has only finitely many isomorphism classes of finite composition factors, then either

(a) $G$ is a finite extension of a direct product of finitely many infinite simple groups, or

(b) $G$ is a finite extension of a residually finite p-group, for some prime $p$.

Proof. Suppose first that $G$ is not residually finite; then $G$ must have a minimal normal subgroup $G_{1}$ of finite index, and $G$ satisfies 
$\min -n$. Since by Theorem A of [7] the property min- $n$ is inherited by normal subgroups of finite index, it follows that $G_{1}$ is an infinite characteristically simple group satisfying $\min n$, and assertion (a) follows.

We therefore suppose $G$ residually finite; we may also assume that $G$ is not a $p$-group, for any prime $p$. We let $p_{1}, \ldots, p_{p}$ be the primes dividing the exponent $e$ of $G$, and write $m_{i}=p_{i}^{-1} e$, for each $i$. Then $\left|G: G^{m}\right|$ is finite for each $i$, and $\left|G: G_{1}\right|$ is finite, where $G_{1}=G^{m_{1}} \cap \ldots G^{m^{r}}$. If $L / M$ is a non-abelian chief factor of $G$, then $G / M$ is finite, and it follows from Lemma $I$ that $G_{I} M / M$ normalizes each direct factor of $L / M$; thus a maximal chain of $G$-subgroups of $G_{1}$ may be refined to a descending chain $C$ of normal subgroups of $G_{1}$ in which all non-abelian factors are simple.

Let $n_{0}$ be the maximal order of a simple non-abelian composition factor of $G$, and let $n=n_{0} !$. The centralizer in $G_{1}$ of each nonabelian simple factor in $C$ contains the intersection $G_{2}$ of all subgroups of index at most $n$ in $G_{1}$; thus $\mathcal{C}$ intersects $G_{2}$ in a descending chain all of whose factors are abelian, and $G_{2}$ is residually soluble. Because $G$ satisfies the maximal condition for normal subgroups, it has only finitely many subgroups of index at most $n\left|G: G_{1}\right|$, by Theorem A of Wilson [8]; therefore $\left|G: G_{2}\right|$ is finite.

We now choose a non-trivial normal subgroup $P$ of $G$, contained in $G_{2}$, such that $P$ is a $\varpi$-group where the set $\varpi$ of primes is as small as possible. We prove that $P$ is a p-group for some prime $p$. Choose any $p \in$ w; by Theorem A of Hall and Higman [2], the p-lengths of the quotient groups $P / N$ with $1 \neq N \triangleleft G$ are bounded, and it follows that $P$ itself has a finite characteristic series all of whose factors are $p$-groups or $p^{\prime}$-groups. Since the smallest non-trivial subgroup $Q$ in this series is normal in $G$ and either a $p$-group or a $p^{\prime}$-group, it 
follows from our choice of $P$ that $P=Q$ and that $P$ is a $p$-group. This completes the proof of Lemma 2.

Proof of Theorem $1(a)$. The hypothesis is that $\underline{\underline{V}}$ is a locally finite variety containing only finitely many isomorphism classes of finite simple groups; Theorem 6 of Kargapolov [3] implies that $\underline{\underline{V}}$ contains no infinite simple groups. If $G$ is an infinite $\underline{=}$-group satisfying max- $n$, then $G$ has a homomorphic image $\bar{G}$ all of whose proper homomorphic images are finite, and we may apply Lemma 2 to deduce that $\bar{G}$ is a finite extension of an infinite $p$-group $P$; and $P$ inherits the condition $\max -n$ from $G$ by Theorem $A$ of [7]. However, locally finite p-groups satisfying $\max -n$ are finite, by Theorem 3.2 of McLain [5], and the result follows.

Proof of Theorem $1(b)$. Suppose that Theorem $1(b)$ is false, and choose a locally finite variety $\underline{\underline{V}}$, with exponent $e$ as small as possible, such that $\underline{\underline{V}}$ contains no infinite simple groups but does contain an infinite group $G$ satisfying $\min -n$. By Theorem A of Wilson [7], we may replace $G$ by its least subgroup of finite index, to suppose that $G$ satisfies min- $n$ and has no proper normal subgroups of finite index. It now follows from our choice of $\underline{\underline{V}}$ that $G$ has no non-trivial homomorphic images of exponent less than $e$.

Suppose that $L / M$ is a chief factor of $G$ which is not locally $p$-soluble, for some prime $p$, and let $N$ be a non-trivial normal subgroup of $L / M$. We write $N^{*}$ for the smallest normal subgroup of $N$ such that $N / N^{*}$ is locally $p$-soluble. Then $N^{*}$ is a subnormal subgroup of $G / M$ having no non-trivial locally p-soluble homomorphic images and so, by Lemma 1 , is normalized by the group $R / M$ generated by all $\left(p^{-1} e\right)$ th powers of elements of $G / M$. Because $G / R$ has exponent less than $e$, we must have $G=R$; thus $N^{*}$ is normal in $G / M$, and either $N^{*}=1$ or $N^{*}=L / M$. But we cannot have $N^{*}=1$, for $L / M$ would then be generated by locally $p$-soluble normal subgroups, and so would be locally p-soluble. Therefore $N=N^{*}=L / M$, and $L / M$ is simple. It follows from our hypothesis on $\underline{\underline{V}}$ that $L / M$ is finite, so that its centralizer in $G$ is a proper subgroup of finite index in $G$. Since this is a contradiction, we conclude that $G$ is locally $p$-soluble for all primes $p$, and so is locally soluble. 
Now suppose that $G$ has elements of prime order $p$. Because $G$ has finite exponent, it follows from Theorem A of Hall and Higman [2] that the $p$-lengths of its finite subgroups are bounded; and because the property "p-soluble of p-length at most $n$ " is a local property, we conclude that $G$ has a finite series each factor of which is either a $p$-group or a $p^{\prime}$-group. So either $G$ has a non-trivial factor group with exponent less than $e$, or $G$ is a locally finite $p$-group of finite exponent satisfying $\min -n$, and so is finite, by Theorem 3.1* of McLain [5]. In either case we have a contradiction, and the proof of Theorem $l$ is complete.

Proof of Theorem 2. We suppose that $\underline{\underline{V}}$ satisfies the hypotheses of Theorem 2 and contains an infinite finitely generated group; then the argument of Baer [1] used in the introduction shows that $\underline{\underline{V}}$ contains an infinite finitely generated group $G$ all of whose proper quotient groups are finite. Now $G$ cannot be a finite extension of a direct product $D$ of finitely many infinite simple groups, because $D$ would also be finitely generated and because $\underline{\underline{V}}$ contains no infinite finitely generated simple groups. Thus, by Lemma $2, G$ is a finite extension of a residually finite p-group $P$, and $P$ is finitely generated. This contradicts hypothesis (iii) of the theorem, and Theorem 2 follows.

\section{References}

[1] Reinhold Baer, "The hypercenter of a group", Acta Math. 89 (1953), 165-208.

[2] P. Hall and Graham Higman, "On the p-length of p-soluble groups and reduction theorems for Burnside's problem", Proc. London Math. Soc. (3) 6 (1956), 1-42.

[3] М.Н. Наргаполов [M.І. Kargapolov], "Локально конечные группы, обладаюин нормальными системами с нонечнымн фанторами" [Locally finite groups possessing normal systems with finite factors], Sibirsk. Mat. ¿̇. 2 (1961), 853-873.

[4] А.И. Костринин [А.І. Kostrikin], "О проблеме Бернсайда" [The Burnside problem], Izv. Akad. Nauk SSSR Ser. Mat. 23 (1959), 3-34; Amer. Math. Soc. Tronst. (2) 36 (1964), 63-99. 
[5] D.H. McLain, "On locally nilpotent groups", Proc. Cambridge Philos. Soc. 52 (1956), 5-11.

[6] John $\mathrm{H}$. Walter, "The characterization of finite groups with abelian Sylow 2-subgroups", Ann. of Math. (2) 89 (1969), 405-514.

[7] John S. WiIson, "Some properties of groups inherited by normal subgroups of finite index", Math. Z. 114 (1970), 19-2l.

[8] John S. Wilson, "Groups satisfying the maximal condition for normal subgroups", Math. 2. 118 (1970), 107-114.

[9] John S. Wilson, "On perfect subnormal subgroups of finite groups", $J$. Algebra 36 (1975), 242-251.

Christ's College,

Cambridge,

England;

Department of Mathematics, Institute of Advanced Studies, Australian National University, Canberra, ACT. 